Cahiers d'études africaines

$223 \mid 2016$

De l'art (d'être) contemporain

\title{
Kouoh, Koyo (dir.). État des lieux. Symposium sur la création d'institutions d'art en Afrique
}

Ostfildern, Hatje Cantz, 2013, 296 p., bibl., ill.

\section{Sophie Eliot}

\section{OpenEdition}

\section{Journals}

Édition électronique

URL : http://journals.openedition.org/etudesafricaines/18565

DOI : 10.4000/etudesafricaines. 18565

ISSN : $1777-5353$

Éditeur

Éditions de l'EHESS

Édition imprimée

Date de publication : 3 octobre 2016

ISSN : 0008-0055

\section{Référence électronique}

Sophie Eliot, «Kouoh, Koyo (dir.). État des lieux. Symposium sur la création d'institutions d'art en Afrique», Cahiers d'études africaines [En ligne], 223 / 2016, mis en ligne le 01 janvier 2016, consulté le 24 septembre 2020. URL : http://journals.openedition.org/etudesafricaines/18565; DOI : https://doi.org/10.4000/

etudesafricaines. 18565

Ce document a été généré automatiquement le 24 septembre 2020.

(c) Cahiers d'Études africaines 


\section{Kouoh, Koyo (dir.). État des lieux. Symposium sur la création d'institutions d'art en Afrique}

Ostfildern, Hatje Cantz, 2013, 296 p., bibl., ill.

Sophie Eliot

\section{KouoH, Koyo (dir.). - État des lieux. Symposium sur la création d'institutions d'art en Afrique. Ostfildern, Hatje Cantz, 2013, 296 p., bibl., ill.}

1 État des lieux... reprend dans une édition bilingue (français et anglais) certaines contributions du Symposium sur la création d'institutions d'art en Afrique, organisé au centre d'art RAW Material Company (RAW) à Dakar, du 18 au 20 janvier 2012, en coopération avec le Goethe-Institut et la Kulturstiftung des Bundes (Fondation culturelle de la République fédérale d'Allemagne). Ce symposium s'intéressait à l'essor et au rôle des initiatives privées et des structures artistiques indépendantes en Afrique. RAW, créé en 2008, en est l'une des manifestations. L'objectif était avant tout de donner la parole aux opérateurs culturels et de proposer un partage d'expériences face aux réalités des scènes artistiques du continent. Le livre offre ainsi un panorama de ces centres indépendants et privés, parmi les plus dynamiques en Afrique et des enjeux qui leur sont associés.

2 Le développement de structures artistiques privées s'intensifie dans le domaine de l'art contemporain en Afrique depuis une dizaine d'années. Il s'agit de participer à la sortie de ce que Simon Njami a nommé l'aphonie de l'Afrique ${ }^{13}$ sur la scène internationale. Les structures présentées dans l'ouvrage assurent une alternative indispensable aux carences, voire aux absences des politiques culturelles et à la dépendance des scènes locales envers les instances artistiques internationales: le symposium s'inscrit ainsi 
dans une volonté d'affirmer un rôle d'acteur et de décideur depuis le continent africain dans les débats contemporains.

3 Les quatre parties du livre - contexte, programmation et orientation, financement et internationalisme, récits - reprennent la ligne du symposium. Les textes décrivent un choix d'expériences en Afrique ainsi que quelques contributions d'opérateurs gérant des structures homologues hors du continent (New York, Bergen, Séoul), apportant ainsi un éclairage comparatif intéressant à un niveau macro et micro, d'une part sur les points communs entre les différents centres et, d'autre part, sur le système dominant de régulation de la visibilité et de la circulation des œuvres ainsi que des discours dits non occidentaux.

4 Dès son introduction, Koyo Kouoh pose la question de l'autonomie du «paysage artistique [en Afrique] après cinquante ans d'indépendance » (p.17) et de la nécessité d'une coopération sud-sud, tout en revenant sur les conditions de la création de RAW liée à l'incapacité de la Biennale de Dakar à remplir l'espace entre deux éditions. On apprend ainsi que l'intitulé RAW Material Company est déjà en soi programmatique de l'activité du centre. En effet, raw material renvoie à l'Afrique comme continent fournisseur de matière première pour les industries mondiales, tandis que company correspond à une approche entrepreneuriale et collective de la production artistique tout en lui apportant un accès à la production et à la transmission de savoirs.

5 Ces lieux sont le produit du désir de leur(s) fondateur(s), comme le soutient Simon Njami dans sa contribution. Il entend prendre ses distances avec l'explication courante qui réduit l'émergence de ces espaces à l'incurie des États africains de mener une réflexion cohérente sur le fait contemporain. Il développe cette idée autour du concept de «bien commun" qu'il emprunte à Jacques Rancière (pp. 22-24). Son propos cependant finit par s'orienter à nouveau vers la question de la dépendance financière du secteur artistique privé africain. Une dépendance qui, malgré la revendication d'autonomie intellectuelle affichée, doit, selon lui, se confronter au paradoxe de la coopération avec les gouvernements respectifs pour assurer des conditions de travail pérennes. Françoise Vergès plaide, quant à elle, pour «une cartographie de l'immatériel »(p.45), revendiquant ainsi une visualisation d'autres temporalités et spatialités, liée au devoir d'y archiver les mémoires orales plus que de reconstruire des discours dominants.

6 Le chapitre sur la programmation et l'orientation des centres privés s'ouvre sur la présentation d'une structure pionnière en Afrique, Doual'art (Douala, 1991), fondée par Didier Schaub (1952-2014) et Marilyn Douala Manga Bell après l'adoption de la loi sur la liberté d'association au Cameroun (1990). D'abord multidisciplinaire, Doual'art se spécialise dans le domaine de l'art contemporain à partir de 1999 et promeut une intervention artistique basée sur une participation citoyenne dans l'espace urbain. Ses fondateurs créent notamment le Salon urbain de Douala, une manifestation triennale, ainsi que des programmations régulières d'expositions et de résidences dans son espace. Un autre catalyseur de la scène artistique de Douala est le centre ArtBakery (Douala, 2003). Il est aujourd'hui tenu par un collectif de cinq personnes, dont Adeline Chapelle et Bill Kouélany qui signent le texte. L'équipe poursuit le travail de son initiateur, l'artiste Goddy Leye (1964-2011), suivant ses mots d'ordre, « engagement, détermination, actions artistiques, pédagogie et avant-garde esthétiques» (pp. 61-62), tout en maintenant « les relations entre art et intérêt collectif» (p. 63) afin d'assurer l'avenir du centre. Car l'un des invariants des divers cas présentés est bien la précarité, 
la difficulté à pérenniser les activités. Les directeurs de ces centres privés sont en recherche constante de nouveaux formats de programmation et de financements adaptés aux restrictions de gérance quotidienne. C'est sur ce terrain de la précarité qu'évolue l'organisation Center for Historical Reenactments (CHR, 2010-2012, Johannesburg) : «Être plus un concept pensant qu'un espace physique » (p.68), un espace "d'extériorisation plutôt que de séjour» (p.69) comme l'écrit l'artiste et commissaire Gabi Ngcobo, co-initiatrice du projet. Elle présente le CHR comme une émanation des limites épistémologiques et institutionnelles de la Biennale de Johannesburg dont la seconde et dernière édition eut lieu en 1997. Le CHR agit comme référent temporel et discursif à la recherche d'engagements alternatifs en calquant sa durée sur l'intervalle de deux ans des biennales.

7 En lien avec cette problématique et l'internationalisation croissante des scènes de l'art, Sarah Rifky, ancienne commissaire à la Townhouse Gallery au Caire, s'interroge sur la négociation en situation de crise, sur la réduction d'acteurs culturels à des régions plutôt que des villes selon l'actualité et surtout sur l'institution comme lieu de vie de l'art. Le centre d'art Beirut (Le Caire, 2012) repose sur un modèle participatif des artistes. La définition de son cadre juridique et administratif a été confiée au duo "Goldin+Senneby ». Quant à la Platform Seoul (Seoul, 2006), il s'agit d'un projet flexible qui change de formats et de thèmes tous les ans, inspiré du modèle de la biennale itinérante Manifesta et qui tente de nouer des relations avec la région qui l'accueille à chaque édition. Sunjung Kim ne conçoit pas Platform Seoul comme un «espace physique, mais [comme] un type de sphère symbolique ou temporaire » (p. 74).

8 «Financements et internationalisme» sont notamment discutés par Yona Backer, fondatrice de la galerie Third Streaming (New York, 2010). Inspirée de la Third Stream Music accordant la musique classique et le jazz, elle travaille selon «le concept de l'hybride et du mélange des genres » (p. 81) tout aussi bien pour la programmation que le financement de son espace, aspirant à de nouvelles formes de collaboration face aux restrictions budgétaires publiques et privées appliquées à la culture aux États-Unis. Plus désenchanté, Juan A.Gaitán remarque la prépondérance de la "diplomatie culturelle » d'organismes de financements pour l'art et la culture situés en Europe de l'Ouest et en Amérique du Nord (p.93) dans le financement des centres d'art privés à travers le monde. Ceux-ci sont, selon lui, « de(s) zones d'autonomie provisoire» (p. 96) mais sont « réduits à fournir des services logistiques de produits culturels [...] ou à soutenir la représentation nationale» (p. 95) de programmes occidentaux capitalistes aux dépens d'une considération des réalités locales.

9 L'ouvrage s'achève sur une série de présentations de témoignages de carrières, intitulée «Récits». Nous retiendrons surtout le texte d'Elvira Dyangani Ose, par ailleurs curator international art à la Tate Modern (Londres), qui rappelle que de nombreux collectifs d'artistes (Laboratoire Agit'art au Sénégal, Kamiriithu Community au Kenya) ont été créés dans la période du Modernisme africain, qu'elle situe à la fin des années 1970 (p. 114), soit des projets similaires aux initiatives contemporaines. Elle présente deux projets : Bessengue City (Douala, 2001-2002) dans le cadre du festival de Doual'art et de Chimurenga Library, archive en ligne de magazines panafricains ${ }^{14}$. Dans ses analyses conclusives, l'auteure pose une série de questions pertinentes sur le statut de l'artiste contemporain dont les stratégies d'«autoreprésentation et [...] [de] reconnaissance » (p. 123) le différencieraient de l'artiste moderne. Et c'est peu dire que 
les évolutions des systèmes institutionnels publics et privés à un niveau local et international y jouent un rôle décisif.

10 État des lieux... propose un tour d'horizon de centres d'art privés principalement africains par ceux qui les gèrent et les dirigent. Par ailleurs, il permet de se familiariser avec la rhétorique des opérateurs culturels dont la présentation répond aussi à un enjeu de promotion et de marketing des structures auxquelles ils sont rattachés. Cependant les contributions des institutions publiques représentées mériteraient une distanciation critique. Leurs discours se confinent à une rhétorique de l'affirmation du dynamisme et de la vitalité qui seraient nouveaux dans les scènes artistiques africaines, prônant une participation à un futur dont elles connaissent très mal le présent. Il serait important de mettre enfin en évidence les interdépendances asymétriques ${ }^{15}$ entre ces institutions et les acteurs culturels africains. Il s'agit de dépendances mutuelles, des centres en Afrique envers les bailleurs de fonds publics - en particulier de la France, l'Allemagne, les Pays-Bas et l'Angleterre - et des institutions envers les acteurs africains afin de maintenir et légitimer leur « diplomatie culturelle » dont ils dictent les règles. Lors de la présentation des Openings Remarks à la Foire d'art contemporain africain 1:54 à Londres en 2014, il fut demandé aux intervenants, notamment à Koyo Kouoh, si ces espaces n'étaient pas un phénomène urbain et si la reproduction du modèle institutionnel occidental n'était pas problématique ${ }^{16}$. Il en ressort que les acteurs du domaine de l'art contemporain africain doivent aujourd'hui encore justifier leur indépendance intellectuelle, culturelle et artistique. Tenir ce symposium en Afrique est, sans aucun doute, une stratégie de visibilité et de prise de parole déterminante de ce que Olu Oguibe nomme les "contested territories": "autonomy", "self-articulation» et "autography» ${ }^{17}$. Poser la question de l'adéquation du modèle institutionnel occidental pour un centre d'art en Afrique, consiste à reprendre l'argument du mimétisme, tenace en art visuel, qui manifeste un manque de connaissances des - et donc de recherches sur les - réalités locales. C'est ignorer les capacités d'adaptation, d'appropriation et d'invention des acteurs culturels africains interagissant au sein d'un système global régulé par des normes d'exclusion et d'inclusion. Les symposiums et publications de ce genre, sans négliger l'interdépendance avec les bailleurs de fonds principalement nord-ouest-européens, sont déterminants pour sensibiliser des auditoires internationaux. Le fait de se positionner dans une logique d'alternative, d'autonomie, de flexibilité, de critique face aux instances de légitimation internationales et locales génère l'invention de solutions " en créant des modèles et plateformes alternatifs pour la négociation de l'art et de l'histoire, la réflexion au sujet de l'archive, la culture visuelle et l'histoire culturelle ", explique Koyo Kouoh (p.17). Des points communs entre les différents centres privés présentés apparaissent : une forte inquiétude pour leur avenir, une conception de l'art comme un bien commun - impliquant partage et participation - et une rupture avec les canons esthétiques et discursifs dominants. Cette présence accrue de lieux alternatifs n'est pas sans rappeler le rôle des Kunstvereine dans la prise d'indépendance de l'art contemporain à l'égard des collectivités locales en Allemagne dans les années 1960-1970.

11 Finalement, certaines questions restent en suspens. Quels sont les outils et les stratégies disponibles pour faire face sur le long terme à des budgets restreints d'autant que les budgets des pays à « diplomatie culturelle » se réduisent? Quels sont les formats envisageables de coopération entre les différentes formes d'institutions publiques et privées aujourd'hui et pour l'avenir? Quels sont les changements 
souhaitables dans les politiques culturelles nationales, étrangères, européennes, africaines ? Comment assurer la longévité de ces espaces sans dépendre de la figure de leur(s) fondateurs(s) ? Quels peuvent être les questions, les défis, les missions à venir pour les acteurs culturels qui prendront la relève? Ces questions auraient pu être discutées dans une conclusion programmatique qui fait défaut à cet état des lieux ${ }^{18}$. Pour d'autres pistes de réflexion et études de cas, le lecteur pourra se reporter à d'autres publications ${ }^{19}$. Il faut néanmoins souligner que la version en français aurait mérité des relectures supplémentaires pour se défaire des nombreuses coquilles et anglicismes. Il aurait été également préférable d'insérer des images illustrant les textes et les activités des centres plutôt que des photographies du symposium, disponibles sur Internet. Malgré ces limites, le livre met en perspective les préoccupations qui sont actuellement au centre du développement culturel et artistique en Afrique.

\section{NOTES}

13. S. NJAMI, "Chaos et métamorphoses", in Africa Remix, catalogue de l'exposition, Paris, Éditions du Centre Pompidou, 2005, pp. 18-19.

14. <http://chimurengalibrary.co.za.>.

15. L. BuccholZ, "Feldtheorie und Globalisierung ", in B. von BismarCK, T. KAUfMANN \& U. WUGGENIG (eds.), Nach Bourdieu. Visualität, Kunst, Politik, Wien, Turia + Kant, 2008, p. 218.

16. <https://www.youtube.com/watch?v=13hI1ieBI3g>.

17. O. OGUIBE, The Culture Game, Minneapolis, University of Minnesota Press, 2004,p. 13.

18. L'éducation des artistes a été le thème de la seconde édition d'«État des lieux", intitulé Symposium sur l'éducation artistique en Afrique, RAW Material Company, Dakar, du 26 au 28 juin 2014 (source : RAW Material Company).

19. Voir E. HARNEY \& D. THOMPSON, « Emerging Platforms for Artistic Production in DRC, Angola and Mozambique », Critical Interventions. Journal of African Art History and Visual Culture, 8 (2), 2014, pp. 133-139 ; B. FISCHER, K. PINTHER \& U.-S. NzEWI (eds.), New Spaces for Negotiating Art (and) Histories in Africa, Münster, Lit Verlag, 2015. 